NÚMERO

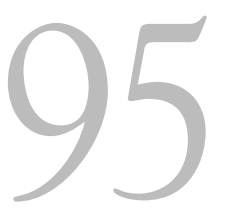

MAYO | AGOSTO [2015]

Miscelánea 


\title{
CRUCE Y DESCRUCE DE MIRADAS AL DERECHO COLABORATIVO
}

\author{
Autor: Luis Bueno Ochoa ${ }^{1}$ \\ Área de Filosofía del Derecho \\ Facultad de Derecho-ICADE \\ Universidad Pontificia Comillas de Madrid
}

\section{Resumen}

El Derecho Colaborativo es un método alternativo de resolución de conflictos. La abogacía colaborativa consiste en una regeneración ética del ejercicio profesional. Un conjunto de miradas, retrospectiva y prospectivamente, conforman una aproximación al Derecho Colaborativo.

Palabras clave: Métodos Alternativos de Resolución de Conflictos; Derecho Colaborativo.

\footnotetext{
${ }^{1}$ lbueno@icade.comillas.edu. El presente artículo, «Cruce y descruce de miradas al Derecho Colaborativo», coincide, sustancialmente, con la Comunicación presentada bajo el mismo título en la Facultad de Derecho de la Universidad Nacional de Educación a Distancia (UNED) el pasado dieciséis de abril de 2015 en las XXV Jornadas de Filosofía Jurídica y Política: Nuevo Derecho, Nuevos Derechos, organizadas por la Sociedad Española de Filosofía Jurídica y Política.
} 


\title{
Cross and uncross glances at Collaborative Law
}

\begin{abstract}
Collaborative Law is an alternative method of conflict resolution. Collaborative Lawyers promote an ethical regeneration practice. A set of looks, retrospectively and prospectively, make an approach to Collaborative Law.
\end{abstract}

Key words: Alternative Dispute Resolution; Collaborative Law.

Recibido: 23-04-2015

Aceptado: 14-07-2015

\section{CRUCE INICIAL DE MIRADAS}

La mirada, marcada por el sentido de la distancia, ofrece una representación. Cuando son varias ofrecen, claro está, varias representaciones. Y en unas Jornadas tituladas «Nuevo Derecho, Nuevos Derechos», esas miradas de las que nos vamos a ocupar van a tener como protagonista al Derecho Colaborativo que, bien mirado, valga el énfasis, constituye un exponente, un claro exponente, aún valdría decir, de un «Nuevo Derecho». No será aventurado comenzar afirmando, por tanto, la consideración del Derecho Colaborativo como Neoderecho. Con posterioridad tendremos ocasión de incidir en esta impresión. Ahora, sin embargo, es momento de ir desvelando qué conjunto de miradas son las que se van a cruzar, y descruzar, en torno al mencionado Derecho Colaborativo.

Serán dos miradas, aunque, en puridad, habría que hablar de dos más, en total, cuatro. Repararemos, no obstante, siquiera sea atendiendo a la propia sistemática de la exposición, en únicamente dos. Y así distinguiremos entre una mirada hacia atrás, llamada retrospectiva, y otra hacia delante denominada prospectiva. Esas dos miradas, sobre las que versarán los dos apartados siguientes, son las que van a conformar el núcleo de la exposición. Esas miradas hacia atrás, primero, y hacia delante, después, empero, se van a ver envueltas, como miradas circulares, en un doble marco con nuevos cruces y descruces que tendrán que ver, por una parte, como queda dicho, con lo nuevo; y, por otra, con lo bueno.

Por lo expuesto hasta el momento tendremos que saber deducir, pues, si del cruce de miradas anunciado, retrospectiva y prospectivamente, resulta que lo nuevo es verdaderamente nuevo; y que lo bueno es realmente bueno. El reflejo de las apariencias y, más exactamente, esa especie de tentativa atávica de confundir, con inspiración cernudiana, deseo y realidad, hará difícil, muy difícil, tomar po- 
sición al respecto. Con todo, como siempre, o como casi siempre, será el tiempo, el paso del tiempo, o mejor, la perspectiva (resultante del conjunto -léase cruce y descruce- de miradas), en concreto, saber mirar con perspectiva, es decir, tener visión de conjunto, lo que propiciará esa aspiración.

En fin, antes de plantearnos dar cumplida respuesta a esa aspiración consistente en indagar acerca del papel que tiene y, sobre todo, que podrá llegar a tener, el Derecho Colaborativo, no deberemos detenernos en consideraciones finales debiendo dirigir nuestros pasos a ese conjunto de miradas: hacia atrás y hacia delante sin dejar de hacer círculos que nos llevarán donde nos tengan que llevar. Dando por hecho que hacia algún lugar, siquiera sea momentáneamente, habrán de llevarnos. Comencemos, pues.

\section{MIRADA RETROSPECTIVA}

Esta primera mirada se va a centrar en desbrozar los diferentes aspectos del Derecho Colaborativo que pasan a relacionarse: origen; contexto; qué es; principios; contenido y ventajas.

\subsection{Origen}

Según José Juan Orbe², el Derecho Colaborativo nace en los años 90 en el Estado de Minnesota (EE. UU.) a impulso de un abogado llamado Stu Webb, especializado en Derecho de Familia, quien cansado de tener que recurrir a los tribunales para resolver los litigios matrimoniales ensayó una nueva fórmula de trabajo que consistió en tomar una drástica decisión que constituye el germen del llamado Collaborative Law: su compromiso consistió en retirarse del caso cuando la vía extrajudicial resultara infructuosa, dejando paso a otro compañero para que fuera éste el que se ocupara de la defensa en la vía judicial. Se trataba, en definitiva, de otra manera de ejercer la profesión de abogado que suscitó interés en otros compañeros hasta el punto que comenzaron a crearse grupos de práctica y reflexión a los que se fueron sumando profesionales de diferentes ámbitos generándose, así, las bases de lo que hoy conocemos como Derecho Colaborativo.

Tras casi diez años de trabajo en la línea comentada, en 1999, se creó la primera asociación que reunió a diferentes profesionales dando lugar al antecedente directo de la International Academy of Collaborative Professionals (IACP); que se ha erigido en un referente ineludible a la hora de aunar los principios de la prác-

${ }^{2}$ Cfr. Orbe, J. J., "El Derecho Colaborativo: un nuevo método de resolución de conflictos”, en Boletín del Ilustre Colegio de Abogados del Señorío de Vizcaya, diciembre 2014, núm. 235, p. 11. 


\section{Luis Bueno OchoA}

tica colaborativa, la formación requerida y los instrumentos y procesos para la buena praxis de este método.

El Derecho Colaborativo se fue extendiendo a Europa y ha llegado hasta nuestro país. Puede destacarse que en Estados Unidos ha evolucionado, como señala Helena Soleto, «como una forma específica de resolución de conflictos, y se ha aprobado incluso una normativa (Uniform Law Collaborative Act, de 2099) por la Conferencia Nacional de Comisionados para la Uniformidad de Leyes Estatales, que ya ha sido adoptada por algunos Estados» ${ }^{3}$.

En España el Derecho Colaborativo ha sido introducido a través de la acción de las asociaciones a partir de 2013. Hay que destacar, a este respecto, por una parte, el papel de la Asociación de Derecho Colaborativo de Euskadi, presidida por María José Anitua (www.derechocolaborativo.es), así como, por otra, el de la Asociación de Derecho Colaborativo de Madrid, presidida por Emiliano Carretero Morales (www.asociacionderechocolaborativo.com).

\subsection{Contexto}

El Derecho Colaborativo está inmerso en eso que se ha venido en llamar el cambio de paradigma que propugna el tránsito de la «justicia confrontativa» a la «justicia del acuerdo» ${ }^{4}$. El contexto, por consiguiente, no es otro sino el de los métodos alternativos de resolución de conflictos (los ADR, como son conocidos por las siglas de la expresión Alternative Dispute Resolution).

El impulso principal, como ha hecho ver la profesora Soleto, lo ha tenido Estados Unidos con la introducción de sistemas de justicia flexibles desde los años 70.

A nivel europeo, hay que remontarse al Consejo Europeo de Tampere de 1999 hasta llegar a la Directiva de mediación civil y mercantil de 2008; la Directiva de víctimas de 2012; la Directiva de mediación en materia de consumo de 2013 y la Reglamentación ODR (Online Dispute Resolution) de 2013.

En España, indudablemente, hay que hacer alusión, cuanto menos, a la Ley 5/2012, de mediación en asuntos civiles y mercantiles, así como a su desarrollo reglamentario (RD 980/2013) y, desde luego, a las incorporaciones previstas en materia concursal a través de la Ley 14/2013, de 27 de septiembre, de apoyo a los emprendedores, sin perjuicio de la transposición -pendiente- de las antedichas Directivas de $2013^{5}$.

${ }^{3}$ Soleto Muñoz, H., "La nueva normativa de mediación y la oportunidad de impulsar una práctica colaborativa del Derecho", en El Notario del Siglo XXI, mayo-junio 2012, p. 19; véase, asimismo, de la precitada autora: «El Derecho Colaborativo como nueva forma de ejercicio», en Iuris, diciembre 2013.

${ }^{4}$ INFORME 2014 DE INNOVASQUE, Agencia Vasca de la Innovación: El retorno de la innovación está en tu mano, "Euskadi pionera en la formación de los profesionales de la justicia del siglo XXI», p. 25; http://www.innobasque.com/Modulos/DocumentosVisor.aspx?.docld=2086/ [Consulta: 06-03-2015].

${ }^{5}$ Cfr. Soleto Muñoz, H., "El Derecho Colaborativo como nueva forma de ejercicio”, op. cit., p. 29. 


\section{3. ¿Qué es?}

Las definiciones del Derecho Colaborativo manejadas van a ser aquellas a las que se remiten en sus respectivos sitios web las dos asociaciones anteriormente traídas a colación.

Así, por un lado, la Asociación de Derecho Colaborativo de Euskadi hace constar que se trata de un «método alternativo de resolución de conflictos innovador, colaborativo y amistoso, pero sobre todo es una nueva forma de entender la defensa jurídica y la justicia desde unos principios y valores». Se busca, ante todo, que el proceso esté centrado «en las necesidades e intereses de las partes, muchas veces ocultos para ellas mismas, separando a la persona del problema, gestionando las emociones y las relaciones entre las partes, a las que se hace partícipes en la búsqueda y adopción de la solución acordada y realmente satisfactoria». Se pone el acento, como base de actuación, en la negociación en equipo, en el que intervienen, por supuesto, abogados, pero también otros profesionales (notarios, economistas, graduados sociales, psicólogos, psiquiatras, pedagogos, coaches, mediadores, árbitros, terapeutas...); y, también, subráyese, en el reconocimiento de las partes como protagonistas en la búsqueda de las soluciones de los conflictos en que se ven envueltas.

Y, por otro lado, digámoslo con cariz complementario, la Asociación de Derecho Colaborativo de Madrid precisa, igualmente, que «el Derecho Colaborativo es un método de resolución de conflictos recientemente introducido en el ejercicio de la abogacía»; añadiendo, a su vez, que «la práctica colaborativa se basa en la firma de un compromiso por las partes y sus respectivos abogados, en que renuncian voluntariamente a enfrentarse en los Tribunales para resolver el conflicto trabajando en común». Se resaltan notas características tales como la voluntariedad y la confidencialidad destacando, finalmente, la visión multidisciplinar del conflicto y, ciertamente, cuál es su finalidad: «Cerrar los conflictos en un único proceso con un acuerdo global, eficaz y ejecutable» ${ }^{6}$.

Antes de abordar los aspectos siguientes convendrá explicitar las diferencias que presenta el Derecho Colaborativo con otros métodos alternativos de resolución de conflictos. La Asociación de Derecho Colaborativo de Euskadi se ocupa de remarcar las diferencias con otros dos en particular: el Arbitraje y la Mediación.

\footnotetext{
${ }^{6}$ Para un adecuado encuadre de la temática de que se trata y conferir, en lo posible, la consistencia necesaria a la doctrina y teoría de la Mediación, véase Orozco Pardo, G. y Monereo Pérez, J. L. (Dirs.) y González De Patto, R. Ma y Lozano Martín, A. M. (Coords.), Tratado de Mediación en la resolución de conflictos, Tecnos, Madrid, 2015; obra en la que se pone de manifiesto que «la Mediación es una práctica necesitada de una sólida construcción teórica» pretendiendo cubrir tres finalidades: la formativa, la informativa y la consulta. Se opta, en suma, por la configuración de un Tratado, es decir, por «la exposición objetiva, integral y ordenada de conocimientos sobre un tópico específico que [...] se subdivide en varios apartados».
} 


\section{Luis Bueno OchoA}

En cuanto al primero, señala que «a diferencia del Arbitraje, que es en definitiva un juez privado, las partes deciden por sí mismas la solución que más les conviene, sin dejar que sea un tercero quien decida por ellas»?

Y en relación con la Mediación, se aclara que «aunque la Mediación tiene muchos aspectos en común, el mediador es y tiene que ser neutral, mientras que los profesionales que intervienen en el proceso colaborativo representan a las partes y requieren determinada cualificación profesional. Tratan de buscar una solución a largo plazo». Por último, se añade que «la mediación requiere de un conflicto previo, cosa que no sucede en el caso del derecho colaborativo, donde su eficacia se incrementa si los acuerdos se planifican con carácter preventivo» ${ }^{8}$.

\subsection{Principios}

Nos haremos eco de los principios enunciados por una y otra asociación de referencia.

La Asociación de Derecho Colaborativo de Euskadi enumera los seis siguientes: trabajo en equipo; buena fe; confidencialidad; transparencia; solución compartida según los intereses de las partes y participación de varios profesionales.

La Asociación de Derecho Colaborativo de Madrid, por su parte, relaciona cinco principios básicos de práctica colaborativa: compromiso de colaboración; método autocompositivo; voluntariedad de las partes; confidencialidad e inhabilitación judicial de los profesionales.

Sendos catálogos de principios ofrecen, por su general coincidencia, una elocuente panorámica acerca del propósito y el eventual alcance que se propone esta nueva forma de ejercicio profesional de la abogacía.

\subsection{Contenido}

Para desbrozar el contenido, o hacernos una idea aproximada pero certera de en qué consiste el Derecho Colaborativo, se ha considerado oportuno repasar el -que se publicita en nuestro país como- primer programa de formación integral

\footnotetext{
${ }^{7}$ Para incidir en la diferenciación entre Derecho Colaborativo y Arbitraje, véase Chillón Medina, J. Ma y Merino Merchán, J. F., Tratado de Derecho Arbitral, Civitas, Madrid, 2014; obra en la que se incluyen las últimas reformas y en la que se hace ver que la Mediación es en muchas ocasiones, como figura que forma parte del conglomerado de sistemas de alternativos de solución de conflictos, la antesala del arbitraje.

${ }^{8}$ Para ahondar en la distinción entre Derecho Colaborativo y Mediación, véase Soleto Muñoz, H. (Dir.) y Carretero Morales, E. y Ruiz López, C. (Coords.), Mediación y resolución de conflictos: técnicas y ámbitos, Tecnos, Madrid, 2013; obra que sirve de guía, tanto en cuanto a las técnicas o estrategias de negociación como en relación con los diferentes ámbitos de aplicación (civil, mercantil, familiar, penal, laboral y comunitario) y en la que se repara, asimismo, en el encaje procesal de la Mediación ante los nuevos retos que la eclosión y la «institucionalización» de la misma está suponiendo en nuestro ordenamiento jurídico.
} 
en Derecho Colaborativo fruto de la colaboración de la Universidad Carlos III de Madrid y la Asociación de Derecho Colaborativo de Madrid.

El propio tenor literal del programa, organizado en cuatro módulos impartidos entre noviembre de 2014 y mayo de 2015, resulta orientativo acerca del desarro1lo, del contenido de la práctica colaborativa. Nos limitaremos a destacar algunas expresiones, o formulaciones, comprendidas en el programa que permitirán conocer en qué se traduce, en la práctica, el objeto de la colaboración; a saber:

La Práctica Colaborativa: el cambio de paradigma... reuniones colaborativas... proceso colaborativo... Negociación colaborativa o negociación por intereses: Modelo Harvard... Los siete elementos de la negociación... La gestión de las emociones en la negociación... Desarrollo de habilidades para la Práctica Colaborativa... Comunicación: escucha activa y asertividad... Enfoque multidisciplinar de la práctica colaborativa. Cómo trabajar en equipo... El papel del abogado colaborativo... del mediador... coach... psicólogo... asesor financiero... otros profesionales...

\subsection{Ventajas}

La mirada retrospectiva, casi como ejercicio de recapitulación, concluye con la enumeración de sus ventajas, siguiendo, nuevamente, el criterio sostenido al efecto por la profesora Soleto: «El abordaje colaborativo de los conflictos, y la exclusión de la resolución por los Tribunales, puede suponer varias ventajas: en primer lugar, un mayor control de las partes sobre el contenido de la resolución del conflicto; en segundo lugar, muy relacionado con lo anterior, la reducción del estrés de las partes vinculado a la falta de control; en tercer lugar, la mayor adecuación de la resolución a los intereses de las partes, y, en cuarto lugar, puede reducir el tiempo de incertidumbre, mejorar la situación de los interesados durante y tras la resolución del conflicto y los costes derivados de su gestión»? ${ }^{9}$.

\section{MIRADA PROSPECTIVA}

La mirada hacia delante, la mirada al futuro, va a ser insinuada, por no decir adivinada, a través de unos cuantos titulares de publicaciones procedentes de fuentes diversas.

Los titulares seleccionados serán, en principio, cuatro; sin embargo, se añadirá un quinto, como colofón; a saber: el Derecho Colaborativo, una vía para resolver

\footnotetext{
${ }^{9}$ Soleto Muñoz, H., "La nueva normativa de mediación y la oportunidad de impulsar una práctica colaborativa del Derecho", op. cit., p. 20; véase, en sentido idéntico, de la misma autora, "El Derecho Colaborativo como nueva forma de ejercicio", op. cit., pp. 30-31.
} 


\section{LuIS BuenO OCHOA}

conflictos; la oportunidad del Derecho Colaborativo; el Derecho Colaborativo, como realidad inminente; el Derecho Colaborativo, una innovadora alternativa y, por último, pronóstico del Derecho Colaborativo.

\subsection{El Derecho Colaborativo, una vía para resolver conflictos ${ }^{10}$}

En efecto, el Derecho Colaborativo irrumpe en nuestro país con un precedente como es el norteamericano: «En Estados Unidos, el 85\% de los conflictos se resuelve por vía no contenciosa. Es decir, por mediación, arbitraje, negociación o gracias al derecho colaborativo, un método alternativo que está comenzando a desarrollarse en España».

El terreno, diríamos, está abonado; queda por ver ahora, y, sobre todo, en el futuro inmediato, si esas expectativas - ¿fundadas?- van a ser correspondidas o si, por el contrario, van a verse defraudadas.

\subsection{La oportunidad del Derecho Colaborativo ${ }^{11}$}

La oportunidad que representa el Derecho Colaborativo es consecuencia, en buena medida, no sólo de las notas características que comparte con otros métodos alternativos de resolución de conflictos; sino de otras que se añaden y dotan al mismo de singularidad. Entre esas notas singulares del Derecho Colaborativo se citan las seis siguientes:

1) Formación (la formación adecuada en negociación pivota en torno al conocido como Modelo Harvard); 2) Multidisciplinariedad (el Derecho Colaborativo exige que los abogados interactúen con otros profesionales tales como, por ejemplo, economistas, arquitectos, ingenieros, mediadores, psicólogos, coaches, etc...); 3) Compromiso ( incluso arriesgada es aquella por la que el abogado colaborativo se compromete a no llevar el asunto a los Tribunales); 4) Respaldo público (es elocuente, en este sentido, el apoyo y respaldo público que la Asociación de Derecho Colaborativo de Euskadi ha obtenido a través de la Dirección de Justicia del Gobierno Vasco y de la Agencia Vasca de Innovación INNOBASQUE); 5) Demanda social (se va extendiendo la opinión de que existe la necesidad o, cuanto menos, el interés latente en nuestra sociedad de arreglar los conflictos fuera de las luchas y contiendas judiciales, «fuera del tatami, fuera del ring», y volver a la satisfacción de resolver los problemas con el otro sin necesidad de llegar a las manos); y

${ }^{10}$ Vargas, M., "Derecho colaborativo, una vía para resolver conflictos", en Expansión, 15-11-2013.

${ }^{11}$ Orbe, J. J., "La oportunidad del Derecho colaborativo", en iHay Derecho? El blog sobre la actualidad jurídica y política: http://hayderecho.com/2014/01/21/la-oportunidad-del-derecho-colaborativo/ [Consulta: 06-03-2015]. 
6) Integración Internacional (el papel que desempeña la International Academy of Collaborative Professionals -IACP- como entidad norteamericana promotora del Derecho Colaborativo pasa por ser la principal instancia en orden a ver confirmado su desarrollo).

\subsection{El Derecho Colaborativo, como realidad inminente ${ }^{12}$}

Que el terreno para el Derecho Colaborativo está abonado empieza ya a ser considerado un lugar común: «Cada vez es más generalizada la percepción del ciudadano español de tener un sistema judicial obsoleto. La lentitud de los procesos, la dilación en la resolución de los mismos, la saturación de los Tribunales, los resultados injustos en muchas ocasiones, y el elevado coste económico [...] hacen que la confianza en la "justicia" sea cada vez menor [...] En definitiva, se nos presenta de manera inminente un novedoso panorama en el ámbito jurídico que favorece el impulso de prácticas colaborativas en beneficio del cliente que evite un proceso judicial incierto, largo y costoso».

\subsection{El Derecho Colaborativo, una innovadora alternativa ${ }^{13}$}

El apoyo institucional al Derecho Colaborativo empieza a vislumbrarse. En este sentido la intervención de Manuel Vallín, Director de Justicia, en un foro como el del Ilustre Colegio de Abogados de Madrid, hace más de un año, el 24 de enero de 2014, para hablar de los parabienes del Derecho Colaborativo hace presagiar ese apoyo. El marco que propicia esta nueva actitud es la inevitable comparación con lo que acontece al otro lado del Atlántico: «El derecho colaborativo lleva desarrollándose más de 20 años en Estados Unidos, donde se negocian un $85 \%$ de los conflictos, llegando a los tribunales el 15\% restante únicamente. En el caso español, un 33\% de los casos se negocian y el 67\% acaba yendo a juicio».

\subsection{Pronóstico del Derecho Colaborativo}

El futuro, o el éxito, del Derecho Colaborativo dependerá de qué sea capaz, finalmente, de ofrecer. José Juan Orbe desde el blog alojado en el sitio web denominado abogacía artesana (www.abogaciaartesana.com), en el que se presenta como Hijo, nieto y sobrino de marinos, se fija en el diagnóstico y en la confianza ${ }^{14}$.

${ }^{12} \mathrm{http}: / / \mathrm{www}$.jenasa.com/juridico/derecho-colaborativo-una-realidad-inminente/ [Consulta: 06-03-2015].

13 IREKIA EUSKO JAURLARITZA-GOBIERNO VASCO, http://www.irekia.euskadi.eus/es/ news/17582-derecho-colaborativo-una-innovadora-alternativa-resolucion-conflictos/ [Consulta: 06-03-2015].

${ }^{14}$ Orbe, J. J., "A gripes y caracoles: apuntes de la ponencia de Derecho Colaborativo y Resolución de conflictos", en http://www.abogaciaartesana.com/derecho-colaborativo/a-gripes-y-a-caracoles-apuntesde-la-ponencia-de-derecho-colaborativo-y-resolucion-de-conflictos/893/ [Consulta: 06-03-2015]. 


\section{Luis Bueno OCHOA}

Así, de un lado, «el papel que puede desarrollar el abogado formado en derecho colaborativo a la hora de encauzar la disputa por un camino u otro puede ser la de ese experto en resolución de conflictos que enviará a su cliente a tribunales cuando considere que ese es el mejor espacio para resolver su disputa o le remitirá a otro tipo de proceso cuando lo considere más adecuado, incluido el derecho colaborativo».

Y, de otro, que «la respuesta que se nos da desde el derecho colaborativo es que lo importante para la elección de este método no está en el tamaño sino en la voluntad de las partes, en el grado de confianza en la apuesta por una solución negociada, en la transparencia, en la confidencialidad, en la búsqueda de mejores soluciones, en el dominio sobre el tiempo y el gasto, y en la profundidad y flexibilidad...».

Para que los presagios no decaigan pueden ser también muy valiosas unas declaraciones recientes de María José Anitua, la Presidenta de la Asociación de Derecho Colaborativo de Euskadi, recogidas en el diario El Correo ${ }^{15}$. Las claves manejadas tienen que ver con la cultura y la filosofía del Derecho Colaborativo. Así pues, por una parte, se trata de «un tema cultural. No se va a solucionar de un día para otro [...] España es el tercer país con más litigios de la OCDE, detrás de Rusia y la República Checa, con todo lo que ello implica de costes económicos, emocionales, empresariales... Nosotros creemos que en pleno siglo XXI existen otras formas de hacer las cosas sin necesidad de llegar a un juicio». Y, por otra, según la filosofía del Derecho Colaborativo, en efecto, el acuerdo «sale más económico que la vía judicial y también es más rápido, ya que en el plazo de seis meses a un año se suele llegar a una solución». Y, además, a las ventajas de ahorro de tiempo y dinero hay que añadir una tercera: «No te dejas la salud como en un proceso judicial».

En esta misma línea de lo futurible, permítasenos decir, convendrá glosar, brevemente, lo que dio de sí el primer Congreso de Derecho Colaborativo organizado por la IACP en Vancouver (Canadá) a finales de 2014. Desde el mencionado blog de abogacía artesana, José Juan Orbe destacaba, en titulares, los tres aspectos siguientes: 1) el reconocimiento del mencionado Congreso como un espacio transnacional y multidisciplinar; 2) la -triple-fijación de las necesidades del cliente en el derecho y práctica colaborativa: procesos, costes y plazos; y, por último, 3) la mención de los tres pilares básicos en la práctica colaborativa: expectativas, confianza y equilibrio ${ }^{16}$. Los tres aspectos mencionados pueden verse complementados, a su vez, con los tres trazos recapitulativos a los que el mismo

15 "Hay otras formas de resolver las cosas sin tener que llegar a juicio", en El Correo, 19-02-2015.

${ }^{16}$ Orbe, J. J., "Compartiendo el Congreso de Derecho Colaborativo de Vancouver", en http://www. abogaciaartesana.com/derecho-colaborativo/compartiendo-el-congreso-de-derecho-colaborativo-devancouver/584/ [Consulta: 06-03-2015]. 
autor se refiere en otra entrada de su blog: uno, el protagonismo del cliente; dos, la función social del abogado y, tres, la necesidad de no olvidar la ética ${ }^{17}$.

La mirada prospectiva del Derecho Colaborativo la vamos a ver culminada por vía de remisión a una muy breve selección de afirmaciones, con el contraste de alguna que otra pregunta, comprendidas en el blog de Amancio Plaza. De esta manera veremos allanado el camino para llegar al apartado final con el que nos proponemos descruzar el cruce de miradas para tratar de lograr ver. Una secuencia provista de pregunta-exclamación-provocación ilustra la conversión al Derecho Colaborativo del prenombrado jurista gallego que se presenta como Navegante, Peregrino, Explorador: «Entonces, ¿te conformas con lo que ya sabes que no funciona, sin darle una oportunidad a escuchar fórmulas innovadoras que acreditan un potencial real de "redefinición de comunidades de intereses”? ¡No me creo que el tan natural "escepticismo” sano, te impida hacer una mínima investigación!» ${ }^{18}$. En otro orden de cosas, nos referiremos sumariamente a la -supuesta- generosidad $^{19}$ del Movimiento del Derecho Colaborativo así como a su previsible - ¿deseable?- expansión ${ }^{20}$.

La generosidad, primeramente, se puede conjugar con el llamado buenismo antropológico, con la «escucha activa» y con el coste de los servicios -que se dicen premium - de la abogacía colaborativa. Sobre el denominado buenismo; es desmentido con contundencia al afirmar que «los abogados implicados en el movimiento de Collaborative Practice implementan no sólo un "proceso colaborativo" en beneficio de los clientes, sino una "ética colaborativa” entre los propios letrados: ¡el letrado de la otra parte es un aliado en el proceso, no el enemigo a batir!». Con la «escucha activa» el abogado no cede, sino que comparte, su protagonismo: «El "paradigm shift" en la función del "abogado colaborativo" es bestial: pasas de "llevar la voz cantante" porque eres el que sabe "de la ley", a tener que "aprender a escuchar" para comprender el conflicto del cliente y sus intereses». Sobre el coste de la abogacía colaborativa no sólo se corrobora el criterio sostenido por Noel Doherty, para quien «el abogado colaborativo no es una ONG sino un prestador de servicios premium»; sino que se añade que «el "proceso colaborativo" debe ser caro: el valor añadido en la superación de conflictos jurídicos computa no sólo

${ }^{17}$ Orbe, J. J., "Presentación del Derecho Colaborativo en Donostia”, en http://www.abogaciaartesana. com/actualidad/presentacion-del-derecho-colaborativo-en-donostia/459/ [Consulta: 06-03-2015].

${ }^{18}$ Plaza, A., "Derecho Colaborativo: una perspectiva del 'año cero' del movimiento del Derecho Colaborativo en España", en http://www.amancioplaza.com/content/derecho-colaborativo-una-perspectiva-del-a\%C3\%B1o-cero-del-moviemiento-del-derecho-colaborativo/ [Consulta: 06-03-2015].

${ }^{19}$ Plaza, A., "La generosidad entre los profesionales del Derecho Colaborativo: una lección desde Cork", en http://www.amancioplaza.com/CPr/la-generosidad-entre-los-profesionales-del-derecho-colaborativo-una-lecci\%C3\%B3n-desde-cork/ [Consulta: 06-03-23015].

${ }^{20}$ Plaza, A., "IACP Forum (Vancouver 2014): un balance descriptivo sobre el 'Movimiento del Derecho Colaborativo' en marcha", en http://www.amancioplaza.com/CPr/iacp-forum-vancouver-2014-unbalance-descriptivo-sobre-el-movimiento-del-derecho-colaborativo-en/ [Consulta: 06-03-2015]. 


\section{Luis Bueno OchoA}

el "resultado material" (cuanto saco) sino la minimización de daños colaterales ( ¿cuánto vale no destruir para siempre una relación comercial, o no dañar-quizás de forma irreversible- el plano personal de los hijos?), el propio aprendizaje en la convivencia del conflicto, y el coste de oportunidad (no pasarte años pendiente de cómo termina todo, o de cuándo se va a reabrir el follón)».

Y, finalmente, la expansión, y la fuerza, del Movimiento del Derecho Colaborativo se conecta con una necesaria regeneración ética en el ejercicio de la abogacía, tal como se desprende de sendas acotaciones que se dirán.

Sobre la expansión, se dice, «está esencialmente vinculada a la máxima profesionalidad de los profesionales intervinientes en el "proceso colaborativo", siguiendo los estándares y protocolos IACP».

Y, sobre la fuerza, se añade que «radica en la convicción de que los seres humanos en conflicto tienen la capacidad de superarlos con la ayuda adecuada, con el apoyo para generar un espacio de seguridad en el que afrontarlos». Liderar el conflicto (Conflict Leadership) es, en suma, como apunta Amancio Plaza refiriéndose a Neil Denny, lo que hay que proponerse hacer-conseguir; y para ello, antes, es imprescindible, hay que convencerse-estar convencido de ello ${ }^{21}$.

\section{DESCRUCE FINAL DE MIRADAS}

Una manera de acometer el tránsito del cruce al descruce de miradas bien pudiera consistir en formular interrogantes. En hacernos preguntas, aclarémoslo, sin necesidad de ofrecer respuestas. Se trata, pues, de motorizar la reflexión tras las miradas hacia atrás y hacia delante a través de diez breves párrafos trufados de preguntas.

Antes de poner «negro sobre blanco» la aludida secuencia de preguntas retomemos, como marco de referencia, lo que habíamos anunciado acerca de esas otras dos miradas que, como podremos comprobar, se acabarán imponiendo, en círculos, y sobre las que se irán posando el grueso de las cuestiones que seguirán. Recordémoslas: una, se fijaba en lo nuevo, y, otra, en lo bueno. Otra forma de referirse al Derecho Colaborativo como Neoderecho, en un caso, y como Derecho ético (o Derecho moral, o Derecho desiderativo..., por no terminar hablando, sin más ni más, de moralismo jurídico), en otro.

¿Es verdaderamente novedoso apostar por la «justicia del acuerdo» en detrimento de la llamada «justicia confrontativa»?

${ }^{21}$ Plaza, A., "Conflict Leadership con Neil Denny: entre la prevención (consultoría de empresa familiar) y la superación (Derecho Colaborativo)", en http://amancioplaza.com/CPr/conflict-leadershipcon-neil-denny-entre-la-prevenci\%C3\%B3n-consultor\%C3\%ADa-de-empresa-familiar-y-la/ [Consulta: 06-03-2015]. 
¿La apuesta por el Derecho Colaborativo es una especie de secuela rousseauniana del «hombre bueno por naturaleza»? ¿Podría en tal caso considerarse un estigma de eso que se conoce como optimismo antropológico?

¿El rechazo al Derecho Colaborativo es una especie de secuela hobbesiana, inspirada en Plauto, del homo homini lupus? ¿Podría en tal caso considerarse un estigma de eso que se conoce como pesimismo antropológico?

¿Sirve el Derecho Colaborativo para «repensar el Derecho»?

¿Con el Derecho Colaborativo el Derecho se hace más y más líquido? ¿Es el Derecho Colaborativo, parafraseando a Zygmunt Bauman, un exponente de eso que podríamos denominar Derecho Líquido?

¿Un Derecho licuado es un Derecho condenado a desaparecer? ¿Hablamos, pues, del paso del estado sólido al líquido para finalmente evaporarse en el gaseoso?

¿La desaparición del Derecho conduciría a una suerte de moralismo jurídico?

Si este Derecho «repensado» acaba con el Derecho, entonces, ihabremos confundido, como si de un delirio se tratase, deseo y realidad?

¿Será exclusivo el Derecho Colaborativo de una sociedad más madura, más dialogante y, en definitiva, más civilizada de la que disfrutamos/padecemos?

Si así fuera, ipara que tenga presente y futuro el Derecho Colaborativo tendremos -itodos?- que tener presente y futuro?

Las miradas al Derecho Colaborativo no terminan, no pueden darse por concluidas, porque, precisamente, es el tiempo, como decíamos al inicio de la exposición, el que irá determinando lo que, recurrentemente, irá dando de sí retrospectiva y prospectivamente. Las miradas en círculos acerca de lo nuevo y lo bueno también nos permitirán ir viendo cuál podrá ser el desenlace. Entretanto, el cruce y descruce de miradas al Derecho Colaborativo nos permiten ver. Eso es: ver lo que vamos viendo y lo que podremos llegar a ver.

\section{BIBLIOGRAFÍA}

ANITUA, M ${ }^{a}$ J., "Hay otras formas de resolver las cosas sin tener que llegar a juicio", en El Correo, 19-02-2015.

CHILLÓN MEDINA, J. M. y MERINO MERCHÁN, J. F., Tratado de Derecho Arbitral, Civitas, Madrid, 2014.

IREKIA EUSKO JAURLARITZA-GOBIERNO VASCO: http://www.irekia. euskadi.eus/es/news/17582-derecho-colaborativo-una-innovadora-alternativa-resolucion-conflictos/ [Consulta: 06-03-2015].

ORBE, J. J., "El Derecho Colaborativo: un nuevo método de resolución de conflictos", en Boletín del Ilustre Colegio de Abogados del Señorío de Vizcaya, diciembre 2014, núm. 235. 


\section{Luis Bueno OchoA}

"La oportunidad del Derecho colaborativo", en ¿Hay Derecho? El blog sobre la actualidad jurídica y política: http://hayderecho.com/2014/01/21/laoportunidad-del-derecho-colaborativo/ [Consulta: 06-03-2015].

"A gripes y caracoles: apuntes de la ponencia de Derecho Colaborativo y Resolución de conflictos", en http://www.abogaciaartesana.com/derechocolaborativo/a-gripes-y-a-caracoles-apuntes-de-la-ponencia-de-derechocolaborativo-y-resolucion-de-conflictos/893/ [Consulta: 06-03-2015].

"Compartiendo el Congreso de Derecho Colaborativo de Vancouver", en http://www.abogaciaartesana.com/derecho-colaborativo/compartiendo-elcongreso-de-derecho-colaborativo-de-vancouver/584/ [Consulta: 06-03-2015].

"Presentación del Derecho Colaborativo en Donostia", en http://www. abogaciaartesana.com/actualidad/presentacion-del-derecho-colaborativo-en-donostia/459/ [Consulta: 06-03-2015].

OROZCO PARDO, G. y MONEREO PÉREZ, J. L. (Dirs.) y GONZÁLEZ DE PATTO, R. Ma y LOZANO MARTÍN, A. M. (Coords.), Tratado de Mediación en la resolución de conflictos, Tecnos, Madrid, 2015.

PLAZA, A., "Derecho Colaborativo: una perspectiva del 'año cero' del movimiento del Derecho Colaborativo en España”, en http:/www.amancioplaza.com/content/derecho-colaborativo-una-perspectiva-del-a\%C3\%B1ocero-del-moviemiento-del-derecho-colaborativo/ [Consulta: 06-03-2015].

"La generosidad entre los profesionales del Derecho Colaborativo: una lección desde Cork", en http://www.amancioplaza.com/CPr/ la-generosidad-entre-los-profesionales-del-derecho-colaborativo-unalecci\%C3\%B3n-desde-cork/ [Consulta: 06-03-23015].

"IACP Forum (Vancouver 2014): un balance descriptivo sobre el 'Movimiento del Derecho Colaborativo' en marcha”, en http://www.amancioplaza.com/CPr/iacp-forum-vancouver-2014-un-balance-descriptivo-sobre-el-movimiento-del-derecho-colaborativo-en/ [Consulta: 06-03-2015].

"Conflict Leadership con Neil Denny: entre la prevención (consultoría de empresa familiar) y la superación (Derecho Colaborativo)", en http://amancioplaza.com/CPr/conflict-leadership-con-neil-denny-entrela-prevenci\%C3\%B3n-consultor\%C3\%ADa-de-empresa-familiar-y-la/ [Consulta: 06-03-2015].

SOLETO MUÑOZ, H. (Dir.) y CARRETERO MORALES, E. y RUIZ LÓPEZ, C. (Coords.), Mediación y resolución de conflictos: técnicas y ámbitos, Tecnos, Madrid, 2013.

SOLETO, H., "La nueva normativa de mediación y la oportunidad de impulsar una práctica colaborativa del Derecho", en El Notario del Siglo XXI, mayo-junio 2012.

"El Derecho Colaborativo como nueva forma de ejercicio", en Iuris, diciembre 2013. 
VARGAS, M., "Derecho colaborativo, una vía para resolver conflictos", en Expansión, 15-11-2013.

VV. AA., Informe 2014 de Innovasque, Agencia Vasca de la Innovación: El retorno de la innovación está en tu mano, "Euskadi pionera en la formación de los profesionales de la justicia del siglo XXI", en http://www.innobasque.com/Modulos/DocumentosVisor.aspx?.docld=2086/ [Consulta: 06-03-2015].

"Derecho colaborativo, una realidad inminente", en http://www.jenasa. com/juridico/derecho-colaborativo-una-realidad-inminente/ [Consulta: 06-03-2015]. 